\title{
Primary T-Cell Lymphoma With Small Intestinal Perforation: A Case Report
}

\author{
Chikae Isayama ${ }^{a}$, Nobuhiro Takeuchi ${ }^{b}$ c, Masanori Takada ${ }^{a}, Y^{\prime}$ Nishida $^{a}$, Yusuke Nomura ${ }^{a}$, \\ Masato Iida ${ }^{a}$, Akihito Otsuka ${ }^{\mathrm{a}}$
}

\begin{abstract}
Gastrointestinal perforation is an emergency condition commonly caused by benign ulcers, diverticulosis, or gastrointestinal malignancies; however, primary intestinal malignant lymphoma (PIML) is a rare etiology. We herein report a case of primary T-cell lymphoma with small intestinal perforation.
\end{abstract}

Keywords: T-cell lymphoma; Small intestinal perforation

\section{Introduction}

Primary intestinal malignant lymphoma (PIML) is most commonly observed in the stomach, followed by the small intestines, colon, and esophagus. It is a relatively rare malignancy, accounting for $1-4 \%$ of all gastrointestinal malignant tumors [1]. Histological examination revealed that almost $90 \%$ of PIML cases are of B-cell origin, whereas primary intestinal Tcell lymphoma is relatively rare [1]. Cases of T-cell lymphoma of the small intestine that were diagnosed following intestinal perforation were rarely reported $[2,3]$. Here, we report a patient with primary T-cell lymphoma accompanied by small intestinal perforation.

\section{Case Report}

An 85-year-old male was brought to the emergency department (ED) of our hospital with a complaint of sudden-onset severe lower abdominal pain. He had a past history of lung tuberculosis and gastric ulcer; the latter was treated with gastrectomy. Four months before he was admitted to ED, he was diagnosed with interstitial pneumonia at another hospital, and

Manuscript accepted for publication November 10, 2016

aDepartment of Internal Medicine, Kawasaki Hospital, Kawasaki, Japan bepartment of Internal Medicine, Kobe Tokushukai Hospital, 1-3-10 Kamitakamaru, Tarumi-ku, Kobe-shi, Hyogo 655-0017, Japan

${ }^{\mathrm{c} C}$ Corresponding Author: Nobuhiro Takeuchi, Department of Internal Medicine, Kobe Tokushukai Hospital, 1-3-10 Kamitakamaru, Tarumi-ku, Kobe-shi, Hyogo 655-0017, Japan. Email: nobuhiro.takeuchi@tokushukai.jp

doi: https://doi.org/10.14740/jmc2690w a $2.5 \mathrm{mg}$ /day dose of prednisolone was initiated. Three months before he was admitted, the patient had an episode of small intestinal obstruction, which was treated conservatively with a nasogastric tube. He suddenly had severe lower abdominal pain and placed a call requesting emergency medical assistance. Upon arrival at the hospital, he repeatedly complained of severe lower abdominal pain. Physical examination revealed an abdomen with board-like rigidity and involuntary guarding. Auscultation revealed moist rales in bilateral lungs. His blood pressure was 115/72 $\mathrm{mm} \mathrm{Hg}$, heart rate was 102 beats/min, and body temperature was $39.6^{\circ} \mathrm{C}$. Blood chemistry analysis revealed severe inflammation with 15,900 white blood cells $/ \mu \mathrm{L}, 83.0 \%$ neutrophils, and $10.1 \mathrm{mg} / \mathrm{dL}$ C-reactive protein; mild anemia with $338 \times 10^{4}$ red blood cells $/ \mu \mathrm{L}$ and $10.7 \mathrm{~g} / \mathrm{dL}$ hemoglobin; and a coagulation defect with $70 \%$ prothrombin and $9.5 \mu \mathrm{g} / \mathrm{mL}$ D-dimer. Further, mildly decreased protein $(5.9 \mathrm{~g} / \mathrm{dL})$ and albumin $(2.5 \mathrm{~g} / \mathrm{dL})$ and mildly increased lactate dehydrogenase $(280 \mathrm{IU} / \mathrm{L})$ and blood urea nitrogen (BUN, $23.3 \mathrm{mg} / \mathrm{dL}$ ) were detected. Tumor markers including carcinoembryonic antigen (CEA) and cancer antigen 19-9 (CA 19-9) were within normal limits. Chest X-ray revealed infiltration shadows in bilateral lower lungs (Fig. 1a), and abdominal X-ray did not detect any free air (Fig. 1b). Non-contrast computed tomography revealed mild infiltration shadows in the bilateral lower lungs (Fig. 2a), a small amount of free air on the surface of the left lobe of the liver (Fig. 2b), and thickening of the jejunal walls (Fig. 2c) with enlarged lymph nodes around the thickened jejunum (Fig. 2d). Based on the radiological findings, perforation of a small intestinal tumor with pneumonia was suspected, and emergency surgery was performed. During surgery, a perforation site was found in the upper jejunum with a tumor surrounded by several enlarged lymph nodes (Fig. 3a). The tumor, including the perforation site, was resected within safe margins (Fig. 3b). Microscopic specimens of the tumor stained with hematoxylin-eosin revealed massive invasion of large-sized atypical lymphatic cells (Fig. 3c, low-power field; Fig. 3d, high-power field). Immunohistochemical staining determined that the tumor specimen was positive for CD45 (Fig. 4a) and CD3 (Fig. 4b) and negative for CD20 (Fig. 4c); these findings were consistent with diffuse T-cell type malignant lymphoma. In addition, immunohistostaining also revealed that $50 \%$ of cancer cells were positive for Ki-67 (Fig. 4d). The postoperative course of this patient was uneventful. On postoperative day 2, sputum examination revealed acid-fast bacilli (Gaffky 7) that led to an additional diagnosis of pulmonary tuberculosis. On postoperative day 5 , the patient was referred to another hospital 

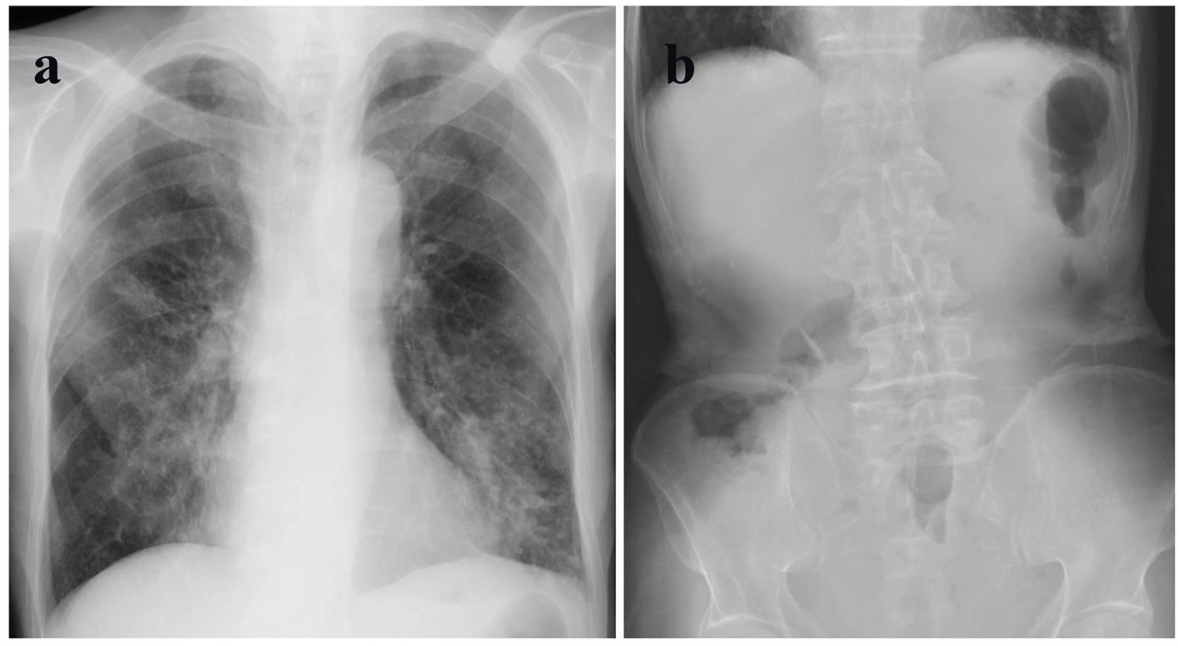

Figure 1. (a) Chest X-ray showing infiltration shadows in bilateral lower lungs. (b) Abdominal X-ray showing the absence of free air.

for chemotherapy for malignant T-cell lymphoma in the small intestines and pulmonary tuberculosis.

\section{Discussion}

Primary gastrointestinal lymphoma (PGL) is a rare condition that rarely affects the small intestine. According to the diagnostic criteria established by Dawson et al [4], PGL should sat- isfy the following conditions: 1) no involvement of superficial lymph nodes, 2) no enlargement of mediastinal lymph nodes by chest X-ray, 3) no abnormal white blood cells in peripheral blood smear, 4) lesion limited to the gastrointestinal tract, and metastasis, if present, limited to the regional lymph nodes, and $5)$ no tumors in the liver or spleen. Based on these criteria, our patient was diagnosed with PGL after the emergency operation.

PGLs account for $30 \%$ of extranodal lymphomas, sec-
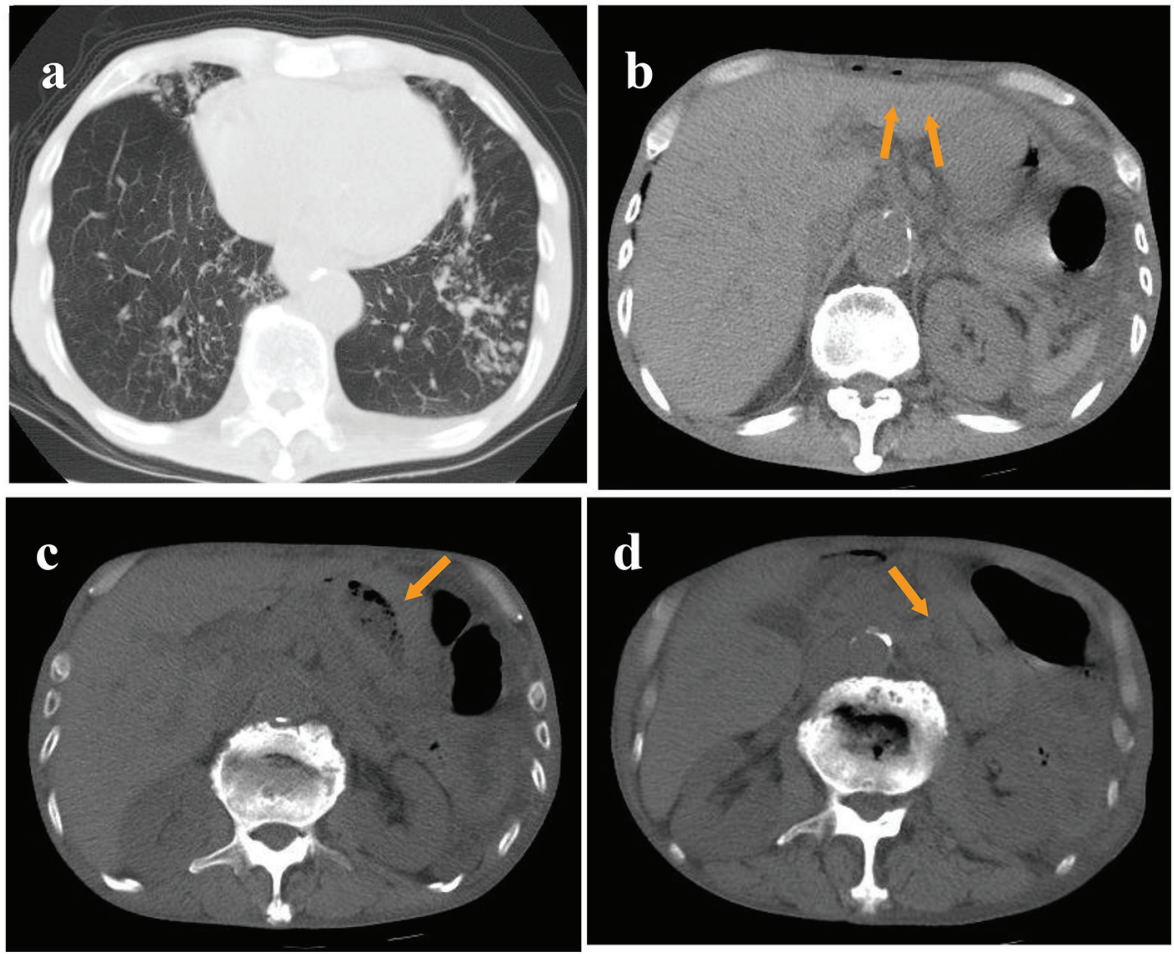

Figure 2. (a) Computed tomography showing mild infiltration shadows in the bilateral lower lungs. (b) A small amount of free air on the surface of the left lobe of the liver. (c) Thickened jejunal walls. (d) Enlarged lymph nodes around the thickened jejunum. 

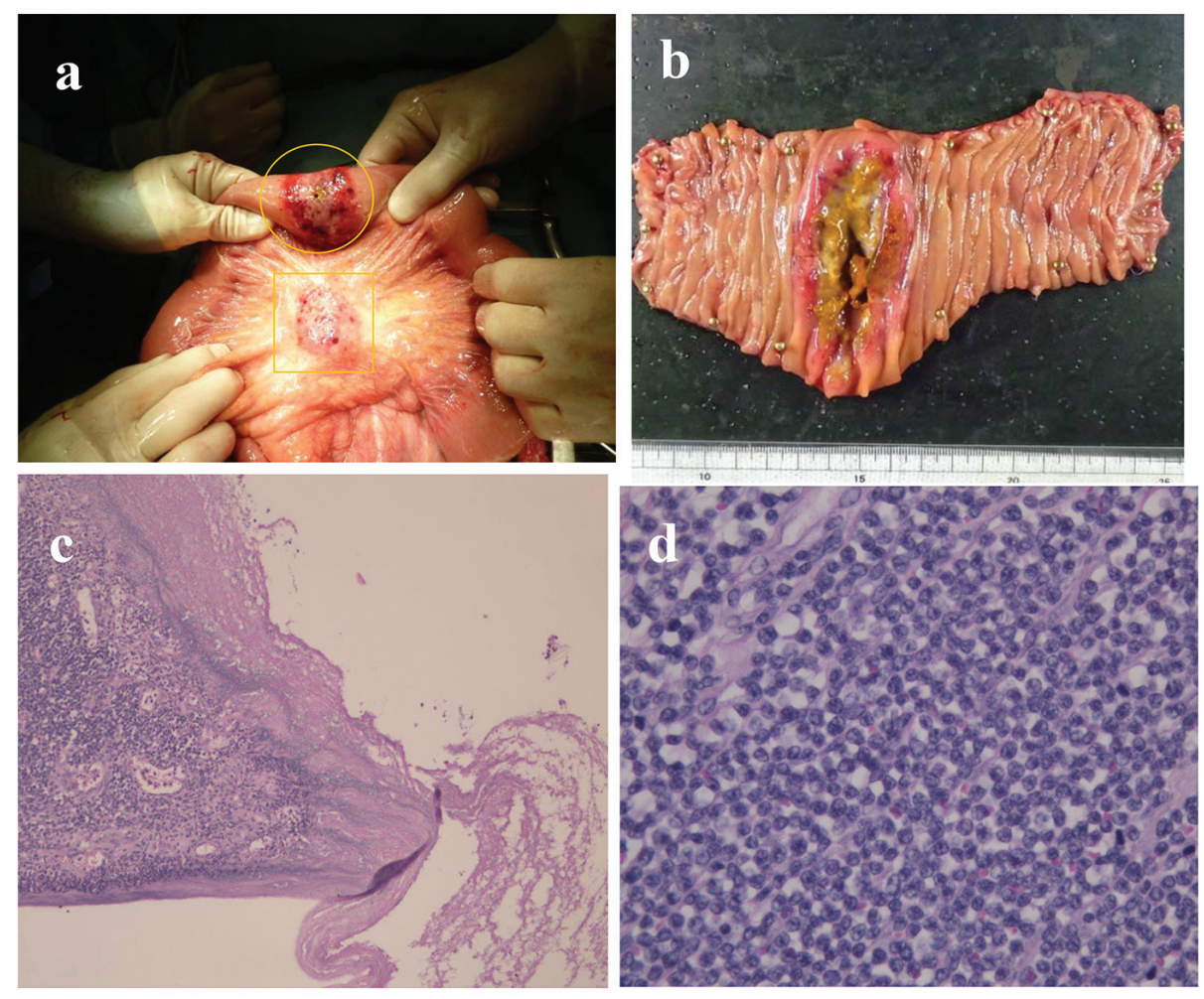

Figure 3. (a) Perforation site located in the upper jejunum (indicated by a circle) with a tumor and several enlarged lymph nodes around the lesion (indicated by a square). (b) Gross specimen of the resected tumors is shown. (c, d) Microscopic specimens stained by hematoxylin-eosin showing massive invasion of large-sized atypical lymphatic cells (c: low-power field; d: high-power field).
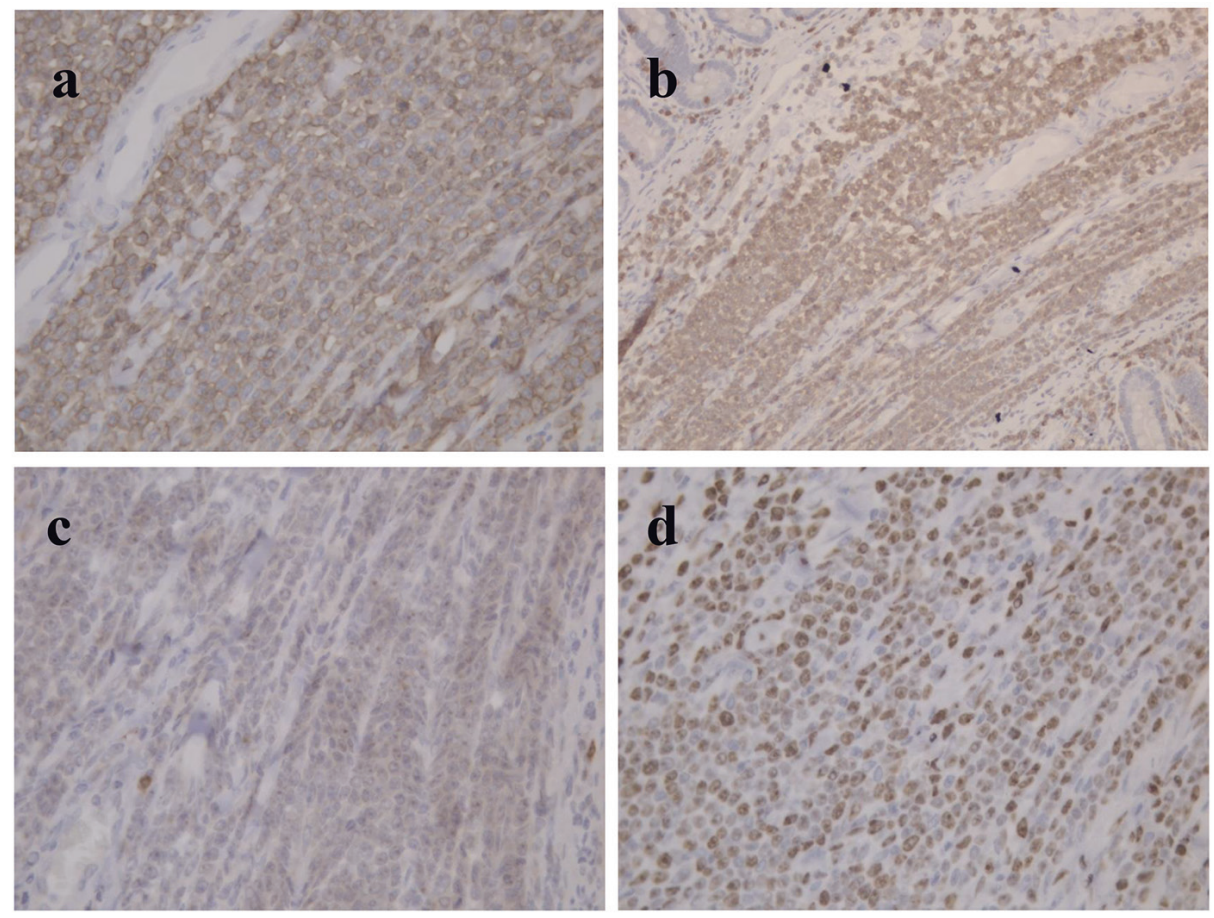

Figure 4. (a, b, and c) Immunohistochemical staining of tumor specimen showing cells positive for CD45 (a) and CD3 (b) and negative for CD20 (c). (d) Immunohistochemical staining for Ki-67 showing $50 \%$ of cancer cells as positive. 
ond most common after the lymphoma of Waldeyer's ring [5]. Within the gastrointestinal tract, PGL affects the stomach, small intestine, and colon in $57-64 \%, 10-31 \%$, and $3-15 \%$ of the cases, respectively [5]. Almost all small intestinal lymphomas are derived from B cells, whereas those with a T-cell origin are relatively rare, accounting for less than $10 \%$ of PGLs [1].

The major symptom of small intestinal lymphoma at presentation is abdominal pain followed by intestinal perforation. Macroscopically, primary small intestinal malignant lymphoma is classified into four types: 1) polypoid, 2) ulcerative, 3) aneurysmal, and 4) constrictive. Among these, the ulcerative type is most prone to aggressive proliferation, which leads to destruction of all intestinal layers with comparatively less connective tissue proliferation, and ultimately, leading to easy perforation.

According to Naqvi et al [6], standardized treatment of PIML includes local resection of the lesion along with lymph node resection in stage I and II patients, and chemotherapy and radiotherapy in stage III and IV patients. Our case was diagnosed as stage III; however, as this patient initially presented with intestinal perforation, emergency surgery preceded chemotherapy.

Shoji et al [7] reviewed 22 cases of perforated T-cell lymphoma of the small intestine; the average age was 53 years, male-to-female ratio was $2: 1$, and the most commonly affected sites were jejunum followed by ileum and duodenum. In some cases, T-cell lymphoma occurs in multiple sites in the intestines, including the duodenum, jejunum, and ileum, which can be attributable to the histological location of T cells; they are found in the outer layers of the mantle zone in lymph nodules that reside in the lamina propria of mucous membranes. T cells proliferate diffusely and progress with mucosal invasion along the Peyer's patch. The prognosis of perforated T-cell lymphoma was reported to be very poor, ranging from 3 days to 15 months, and all reported cases underwent surgical resection followed by postoperative chemotherapy. Prognostic factors in PIMLs are determined by clinical staging, immunophenotyping of T- and B- cells, and the extent of resection. Intestinal perforation is associated with worse prognosis; thus, proper diagnosis and treatment before perforation is desired for improved prognosis. Our patient presented with small intestinal obstruction 3 months before the perforation, and the underlying cause was not known. Further examination and a more thorough discussion to explore the etiology at that time would have allowed earlier detection of the small intestinal lymphoma. It should also be noted that administration of prednisolone without proper diagnosis most likely deteriorated pulmonary tuberculosis as well as small intestinal ulceration, which might have resulted in its perforation.

\section{Conclusion}

We herein report a patient with primary small intestinal T-cell lymphoma with intestinal perforation as the presenting symptom. Albeit rare, PIMLs may present with intestinal perforation, and their early diagnosis is critical due to poor prognosis in cases with perforation.

\section{References}

1. Freeman C, Berg JW, Cutler SJ. Occurrence and prognosis of extranodal lymphomas. Cancer. 1972;29(1):252260.

2. Kim YS, Choi YS, Park JS, Kim BG, Cha SJ, Chi KC, Park SJ, et al. Case of small bowel perforation due to enteropathy-type T-cell lymphoma. Yonsei Med J. 2009;50(6):859-861.

3. Matsumoto K, Chikumi Y, Otani H, Hara A, Maeda K, Yasugi A, Murawaki Y, et al. [Two cases of primary T cell lymphoma of the small intestine diagnosed by perforated peritonitis]. Nihon Shokakibyo Gakkai Zasshi. 2007;104(3):388-393.

4. Dawson IM, Cornes JS, Morson BC. Primary malignant lymphoid tumours of the intestinal tract. Report of 37 cases with a study of factors influencing prognosis. Br J Surg. 1961;49:80-89.

5. Yao T. Primary small intestinal tumors. Stomach and Intestine. 2001;6:871-881.

6. Naqvi MS, Burrows L, Kark AE. Lymphoma of the gastrointestinal tract: prognostic guides based on 162 cases. Ann Surg. 1969;170(2):221-231.

7. Shoji W, Takahashi M, Naito H. A Case of Primary T-cell of the Small Intestine with Perforated Peritonitis. J Jpn Surg Assoc. 2011;72(3):710-715. 